\title{
Pelvic Hematoma Due to Anticoagulant Therapy Causing Urethro-Vesical Anastomosis Disruption After Radical Prostatectomy: A Case Report
}

\author{
Madhuri Kashyap ${ }^{\mathrm{a}}$, Alfredo Harb-De la Rosa ${ }^{\mathrm{a}}$, Mark Solowaya, \\ Murugesan Manoharan ${ }^{\mathrm{a}, \mathrm{b}}$
}

\begin{abstract}
Pelvic hematomas can form as a consequence of anticoagulant therapy after pelvic surgeries such as radical prostatectomy (RP). This can cause a significant disruption of the urethro-vesical anastomosis. We report a case of a 58-year-old male who presented with prostate cancer (PC) and underwent RP. Post-operatively, he developed deep vein thrombosis (DVT) and pulmonary embolism (PE). He was started on anticoagulant therapy which caused a pelvic hematoma resulting in the urethro-vesical anastomosis (UVA) disruption.
\end{abstract}

Keywords: Pelvic hematoma; Urethro-vesical anastomosis; Disruption; Radical prostatectomy

\section{Introduction}

Radical prostatectomy (RP) is a commonly performed curative procedure for localized prostate cancer (PC). The incidence of DVT ranges from $1 \%$ to $5 \%$ in men with $\mathrm{PC}$ undergoing RP [1]. Anticoagulant therapy for deep vein thrombosis (DVT) might lead to the development of pelvic hematoma which may be of no consequence in the majority of the patients. However, in rare occasions, large hematomas following the administration of anticoagulants can cause a significant disruption of the urethro-vesical anastomosis (UVA). We report a case where significant post-operative hematoma resulted in complete UVA disruption. Management of this case is described below.

Manuscript accepted for publication September 12, 2014

${ }^{a}$ Department of Urology, University of Miami Miller School of Medicine, Miami, FL, USA

${ }^{\mathrm{b}}$ Corresponding Author: Murugesan Manoharan, Department of Urology, P.O. Box 016960 (M814), University of Miami Miller School of Medicine, Miami, FL 33101, USA.

Email: mmanoharan@med.miami.edu

doi: http://dx.doi.org/10.14740/wjnu183w

\section{Case Report}

A 54-year-old African American male, presented to our facility with a prostate specific antigen (PSA) of $5.8 \mathrm{ng} / \mathrm{mL}$. Prostate biopsy revealed three cores of Gleason 6 adenocarcinoma. On digital rectal examination he was found to have an approximately $60 \mathrm{~g}$ prostate, which was firm and smooth. He underwent an open retropubic radical prostatectomy (RRP). The pathologic specimen showed Gleason 7 adenocarcinoma occupying $20 \%$ of the specimen. The stage was T1cN0M0. On the fourth post-operative day, the patient presented to the Emergency Department in another facility complaining of chest pain and shortness of breath. A clinical diagnosis of pulmonary embolism (PE) was made and he was treated with a full dose of IV heparin. He subsequently developed hematuria and complained of lower abdominal pain. On physical exam, he was found to be pale, with diffuse abdominal tenderness, guarding and tachycardia. The patient was then transferred to the Surgical Intensive Care Unit (SICU) at our facility. He received two units of packed red cells. A cystogram showed complete disruption of the UVA with displacement of the bladder neck in the left upper quadrant of the abdomen producing a "pie in the sky" appearance. However, there was no evidence of urinary extravasation indicating an intact bladder neck (Fig. 1, 2). A CT scan revealed a very large pelvic hematoma displacing the bladder neck towards the left upper quadrant (Fig. 3). An IVC filter was inserted to prevent migration of thrombi so that he could discontinue the use of anticoagulants. Patient was managed conservatively for 6 - 8 weeks with gentle trac-

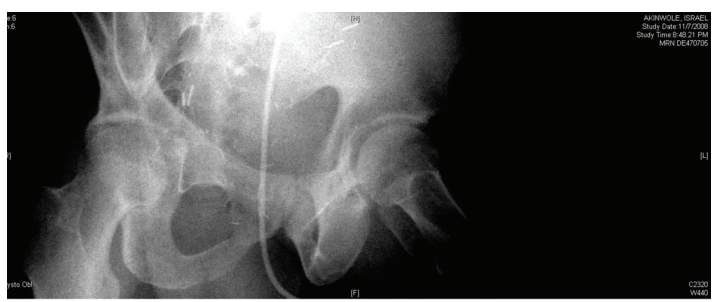

Figure 1. Showing "pie in the sky" appearance of the bladder base with intact bladder neck and no urinary extravasation. 


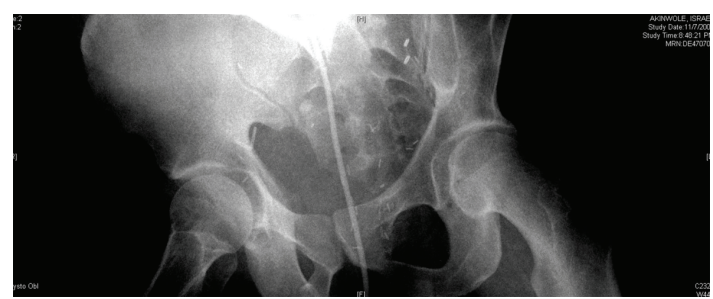

Figure 2. Showing significant displacement of the bladder neck (oblique view).

tion, and this helped maintaining the bladder neck within the pelvis. A cystogram showed no extravasation of urine. The Foley catheter was removed. The patient voided well initially; however, he developed bladder neck contracture (BNC) after 2 weeks. He was managed with dilation of the bladder neck, but the bladder neck did not return to the pelvis. It was found to be $2 \mathrm{~cm}$ away from the urethra, and a "pseudotunnel" made of scar tissue was seen between the true bladder neck and the membranous urethra. A surgical approach was used to explore the patient and the "pseudo-tunnel" was resected. Re-anastomosis of the bladder neck with the urethra was performed. The patient has remained continent since then and voids with minimal post void residual urine. There has been no recurrence of BNC (Fig. 4, 5). He needs intra-cavernous injections to help with erections. His postoperative PSA has remained undetectable.

\section{Discussion}

Patients undergoing RP are at a higher risk to develop thromboembolic events [2]. It is a routine practice to use prophy-

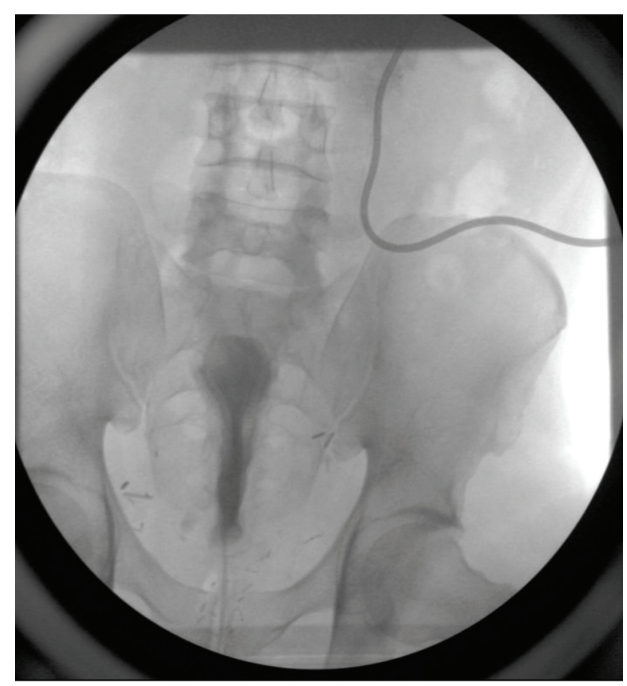

Figure 3. It shows pre-operative cystogram showing cephalic displacement of the bladder neck, and compression of the bladder.

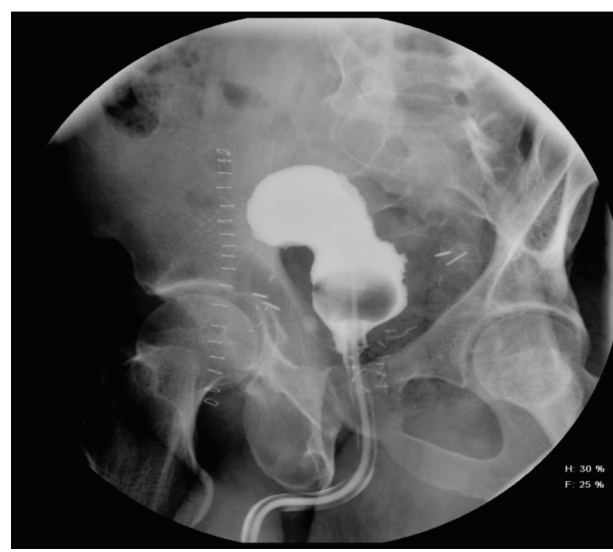

Figure 4. It shows post-operative pericatheter urethrogram with normal bladder neck and urethra.

lactic heparin to prevent DVT. However, early mobilization and the use of mechanical compression have shown to result in a low incidence of DVT, obviating routine prophylaxis with heparin [3]. In this case, a therapeutic dose of heparin was administered to treat PE. This resulted in a significant amount of pelvic hematoma, which is a disruption in UVA. O'Malley et al found in their study that the volume of the para anastomotic hematoma is a predictor of anastomotic extravasation [4]. In our case, CT scan showed no extravasation and the bladder neck was found to be competent. BNC could initially be managed endoscopically [5-7]. If the conservative method of treatment fails, the patient could be managed surgically, as in our case $[8,9]$.

\section{Conclusion}

This case report illustrates the development of pelvic hematoma and its subsequent complications as a consequence of anticoagulant therapy soon after RP. While most of the BNC

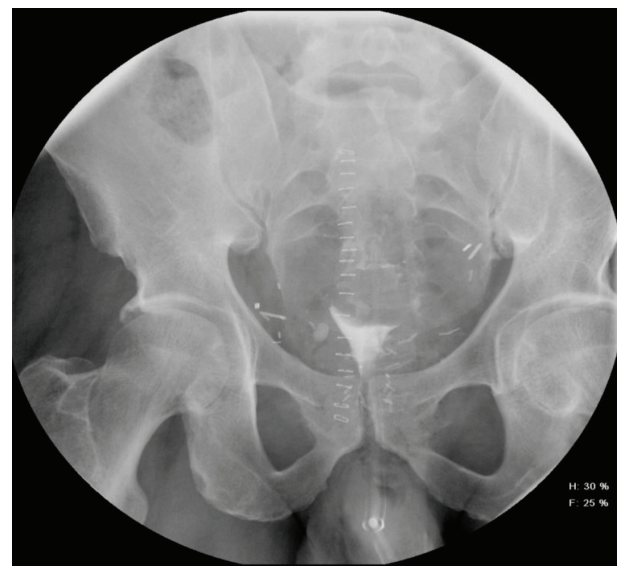

Figure 5. Post-drainage film showing absence of extravasation. 
that result in such a complication could be treated endoscopically, our case required an open intervention which is rare. It is rewarding to see that the patient has remained asymptomatic.

\section{References}

1. Beyer J, Wessela S, Hakenberg OW, Kuhlisch E, Halbritter K, Froehner M, Wirth MP, et al. Incidence, risk profile and morphological pattern of venous thromboembolism after prostate cancer surgery. J Thromb Haemost. 2009;7(4):597-604.

2. Van Hemelrijck M, Garmo H, Holmberg L, Bill-Axelson A, Carlsson S, Akre O, Stattin P, et al. Thromboembolic events following surgery for prostate cancer. Eur Urol. 2013;63(2):354-363.

3. Koya MP, Manoharan M, Kim SS, Soloway MS. Venous thromboembolism in radical prostatectomy: is heparinoid prophylaxis warranted? BJU Int. 2005;96(7):10191021.

4. O’Malley RL, Telegrafi S, Laze J, Lepor H. Para-anastomotic haematoma volume predicts the presence of anas- tomotic extravasation after radical retropubic prostatectomy. BJU Int. 2010;105(1):34-36.

5. Popken G, Sommerkamp H, Schultze-Seemann W, Wetterauer U, Katzenwadel A. Anastomotic stricture after radical prostatectomy. Incidence, findings and treatment. Eur Urol. 1998;33(4):382-386.

6. Gousse AE, Tunuguntla HS, Leboeuf L. Two-stage management of severe postprostatectomy bladder neck contracture associated with stress incontinence. Urology. 2005;65(2):316-319.

7. Yurkanin JP, Dalkin BL, Cui H. Evaluation of cold knife urethrotomy for the treatment of anastomotic stricture after radical retropubic prostatectomy. J Urol. 2001;165(5):1545-1548.

8. Wessells H, Morey AF, McAninch JW. Obliterative vesicourethral strictures following radical prostatectomy for prostate cancer: reconstructive armamentarium. J Urol. 1998;160(4):1373-1375.

9. Simonato A, Gregori A, Lissiani A, Carmignani G. Twostage transperineal management of posterior urethral strictures or bladder neck contractures associated with urinary incontinence after prostate surgery and endoscopic treatment failures. Eur Urol. 2007;52(5):14991504. 